\title{
Cambios del pH salival por el consumo de jugos de frutas industrializados y su efecto en la salud gingival en niños de la Institución Educativa "Isabel La Catòlica"
}

\author{
Variations in salivary $\mathrm{pH}$ due to the consumption of industrialized fruit juices \\ and its effect on gingival health in children of the Educational Institution \\ "Isabel La Catòlica"
}

Ysla-Cheé R. ${ }^{1 a}$, Pareja-Vásquez M. ${ }^{1 b}$

\section{RESUMEN}

Objetivo: Determinar la relación entre la variación del pH salival por el consumo de jugos de frutas industrializados y la salud gingival en niños de la Institución Educativa (I.E.) "Isabel La Católica". Material y Método: Es un estudio observacional, analítico de corte transversal. Se evaluaron 108 niños de ambos sexos, con edades de 6 a 8 años. La muestra fue dividida en dos grupos, un grupo de estudio que bebía jugos de frutas industrializados y un grupo control, que bebía agua. Se realizó una medición basal del $\mathrm{pH}$ salival para ambos grupos con un $\mathrm{pH}$ metro digital, y después de beber el jugo de frutas industrializado o el agua, se volvió a medir a intervalos de 5 y 10 minutos. La condición gingival fue evaluada mediante los Índices Gingival de Löe Silness e Indice PMA. Resultados: No se encontró correlación entre el índice gingival de Löe Silness y el $\mathrm{pH}$ salival en ambos grupos, $\mathrm{p}>0.05$. En el grupo de estudio el $\mathrm{pH}$ salival basal fue ligeramente ácido y se observó una mayor caída del pH a los 5 minutos de consumir el jugo de frutas, $p<0.001$. Se halló una correlación inversa entre el Índice gingival y el pH salival en el grupo control, a mayor índice gingival menor $\mathrm{pH}$ salival. El índice gingival en el grupo de estudio fue moderado y en el grupo control leve. Conclusiones: No hay correlación entre la salud gingival de los niños evaluados y el pH salival asociado al consumo de jugos de frutas industrializados.

Palabras claves: pH; Saliva; Jugos de frutas; Índice gingival; niño.

\section{ABSTRACT}

Objective: Objective: To determine the relationship between the variation of salivary $\mathrm{pH}$ by the consumption of industrialized fruit juices and gingival health in children of the Educational Institution (I.E.) "Isabel La Católica". Material and Method: This is an observational, analytical, cross-sectional study. We evaluated 108 children of both sexes, aged 6 to 8 years. The sample was divided into two groups, a study group that drank industrialized fruit juices and a control group that drank water. A basal salivary $\mathrm{pH}$ measurement was performed for both groups with a digital $\mathrm{pH}$ meter, and after drinking the industrialized fruit juice or water, it was measured again at intervals of 5 and 10 minutes. The gingival condition was evaluated by Löe Silness Gingival Index and PMA Index. Results: No correlation was found between the Löe Silness Gingival Index and the salivary pHin both groups, $\mathrm{p}>0.05$. In the study group, basal salivary $\mathrm{pH}$ was slightly acidic and a greater fall in pH was observed after 5 minutes of consuming fruit juice, $\mathrm{p}<0.001$. An inverse correlation was found between the gingival index and the salivary $\mathrm{pH}$, in the control group, with a higher gingival index, lower salivary $\mathrm{pH}$. The gingival index in the study group was moderate and in the mild control group. Conclusions: There is no correlation between the gingival health of the children evaluated and the salivary $\mathrm{pH}$ associated with the consumption of industrialized fruit juices.

Key words: pH; Saliva; Fruit juices; Gingival Index; Child.

Recibido: $\quad 20$ de mayo de 2018

Aprobado: 28 de setiembre de 2018

Publicado: 30 de octubre de 2018

1 Universidad de San Martin de Porres. Lima, Perú.

a Doctora en Odontología. Docente de Periodoncia.

b Doctora en educación. Profesora asociada de periodoncia.

Correspondencia:

Rosa Elizabeth Ysla Cheé

Correo electrónico: rosayslache@yahoo.es
Este es un artículo Open Access distribuido bajo la licencia Creative Commons Atribución-NoComercialCompartirlgual 4.0 


\section{INTRODUCCIÓN}

La elevación en el consumo de jugos de frutas industrializados especialmente entre los niños es de gran preocupación, principalmente porque se reportan estudios que relacionan este consumo con la erosión y caries dental (1-3).

Se asume que la ingesta de jugos de frutas industrializados influye sobre el $\mathrm{pH}$ salival y esta condición a su vez repercutiría sobre los tejidos dentales y también gingivales.

Se debe considerar que la saliva juega un rol importante en el mantenimiento de la integridad de los dientes por la acción buffer, a la vez que es un fluido importante porque actúa como un mecanismo defensivo para prevenir la formación de caries y enfermedad periodontal. La velocidad y cantidad de flujo salival, que favorece la limpieza de sustratos bacterianos y protege las superficies bucales ${ }^{(4)}$

La saliva tiene además propiedades antibacterianas que se originan de factores inmunes, que le dan un poder anticariogénico, a la vez posee una capacidad amortiguadora y neutralizadora de los ácidos que producen las bacterias cariogénicas o que son ingeridos en la dieta, esto además le permite mantener un $\mathrm{pH}$ constante, a la vez que es una fuente de calcio y fosfato, requeridos para la remineralización del esmalte dental(5). La saliva en condiciones de salud bucal, tiene un $\mathrm{pH}$ que oscila entre 7 a 7.4, lo que indicaría que el grado de acidez o alcalinidad estaría equilibrado. Sin embargo, este puede disminuir al ingerir alimentos con ph ácido o bebidas con carbohidratos fermentables. Cuando el $\mathrm{pH}$ salival disminuye, es decir se hace más ácido, y además hay una microbiota cariogénica, ingesta de carbohidratos, presencia de un hospedero susceptible y se mantiene en esta condición durante mucho tiempo, pueden formarse caries, especialmente a nivel cervical así como manchas blancas en el esmalte. Las caries a nivel cervical se asocian con la formación de recesiones gingivales (6).

Diversos estudios reportan que en presencia de un pH salival más ácido hay mayor prevalencia de caries dental (6-9,), por ello es importante el estudio de alimentos o bebidas que se asocien a la disminución del $\mathrm{pH}$ salival.

El consumo de jugos de frutas pueden alterar el pH salival (10) por lo que podría considerarse un factor extrínseco. ${ }^{(10)}$. Su consumo ha sido relacionado a procesos de caries y erosión del esmalte, más aun por la gran cantidad de azúcares que contienen ${ }^{(1,2)}$. Estudios como el de Goel Isha et al. reportan que los jugos de frutas pueden llevar a un ph salival más bajo incluso que las bebidas carbonatadas, ellos realizaron un estudio en 39 niños entre 8 a 12 años, para evaluar el $\mathrm{pH}$ salival en intervalos de tiempo, después del consumo de jugo de frutas y bebidas carbonatadas, mostraron que los valores del $\mathrm{pH}$ después del consumo de ambas bebidas disminuían, con las bebidas carbonatadas obtuvieron un valor medio de $5.47 \pm 0.78$; y después del consumo de jugos de frutas una media de $4.89 \pm 0.97$ hubo una mayor caída en el pH salival en el caso del consumo jugo de fruta en comparación con las bebidas carbonatadas ${ }^{(11) .}$

Por otro lado, diversos estudios epidemiológicos indican que la prevalencia de enfermedad periodontal se observa desde la primera infancia. Casos de gingivitis suelen observarse en niños y estos tienden a aumentar con la edad, comienzan en los dientes deciduos y alcanzan su pico en la pubertad (12-16).

Sobre la relación entre las variables condición gingival y $\mathrm{pH}$ salival puede citarse el estudio realizado por Ahlam T.et al. en una población de 192 sujetos (96 niños de 4 a 5 años y 96 adultos de 23 a 25 años), en el cual encontraron relación entre la variación del ph salival y la presencia de gingivitis, placa dental y caries dental. Ellos describen una correlación positiva entre el Índice gingival y el ph salival, una correlación negativa entre el ph salival y la presencia de caries dental, y finalmente encuentran que al aumentar las puntuaciones del Índice de placa el ph salival disminuye. Además, observaron que el $\mathrm{pH}$ salival era más ácido en la población de niños que en la de adultos (17). Ysla et al. (2006) por el contrario, halló en su estudio con niños con Síndrome de Down de 6 a 8 años, comparado con un grupo control, un $\mathrm{pH}$ salival ligeramente alcalino $7.96+0.36$. No encontró asociación entre los valores del $\mathrm{pH}$ salival y las condiciones del estado periodontal. Además observó, que el $92.9 \%$ de niños presentó gingivitis leve(15).

La literatura sobre el efecto del consumo de jugos de frutas industrializados en la salud oral es insuficiente y más sobre los efectos del $\mathrm{pH}$ salival por consumo de estas bebidas, en la salud gingival de los niños.

El objetivo del estudio fue conocer cuál es el efecto en la salud gingival de los niños de la Institución Educativa "Isabel La Católica", de los cambios del pH salival, por el consumo de jugos de frutas industrializados.

\section{MATERIAL Y MÉTODO}

El presente estudio es observacional, descriptivo, analítico de diseño prospectivo de corte transversal. La muestra estuvo constituida por 108 niños de ambos sexos, de 6 a 8 años de la I.E. Isabel la Católica. Fueron divididos en dos grupos, un grupo de 
de estudio que bebía jugos de frutas industrializados y un grupo de control que bebía agua embotellada. Se coordinó el desarrollo del estudio con el Director de la Institución Educativa "Isabel La Católica". Posteriormente se informó a los padres o apoderados las características del estudio y se les solicitó que firmaran el consentimiento informado si estaban de acuerdo en la participación de sus niños. Este estudio fue aprobado por el Comité de Ética en Investigación de la Facultad de Odontología de la Universidad de San Martín de Porres.

Se seleccionó para la muestra a los niños, cuyos padres hubieran firmado el consentimiento informado y que voluntariamente aceptaran participar en este estudio. Se excluyó a los niños que estuvieran recibiendo tratamiento sistémico, que hubieren recibido tratamiento con antibióticos/antinflamatorios desde hace tres meses, aquellos con impedimentos para realizar una higiene bucal adecuada y con tratamiento de ortodoncia.

El examen clínico fue realizado por un solo examinador, especialista en periodoncia. Utilizó para el examen luz artificial y empleó una sonda periodontal de la OMS, marca Hu-friedy (USA). Se evaluó la condición gingival de los niños, mediante los Índices gingivales PMA y de Löe Silness. Para evaluar la presencia de placa dental se utilizó el Índice de Higiene Oral Simplificado.

Para medir el pH salival se solicitó a cada niño que se sentara cómodamente y después se recolectó una muestra de su saliva no estimulada, en un vaso descartable previamente enjuagado con agua destilada y calibrado en milímetros. Este proceso se realizó en horas de la mañana. Se realizaron tres mediciones: una basal (antes de beber el jugo de frutas industrializado o el agua), una a los 5 y otra a los 10 minutos después de ingerir una de las bebidas. Para la medición del $\mathrm{pH}$ salival se empleó un $\mathrm{pH}$ metro digital marca "Hanna" modelo HI98107 (China), calibrado debidamente. El electrodo del peachìmetro fue introducido en la saliva y luego se hizo la lectura. Entre cada medición se enjuagó el electrodo con agua destilada.

La información fue registrada en fichas y los datos obtenidos fueron procesados y analizados con el paquete estadístico SPSS versión 18.

Las diferencias entre los grupos para las variables $\mathrm{pH}$, salud gingival, se realizaron utilizando la prueba de Friedman, U de Mann Whitney con un nivel de significancia del $5 \%$.
Se efectuaron cálculos de estadísticos descriptivos y para establecer las comparaciones entre grupos se utilizó la correlación RhO de Spearman.

\section{RESULTADOS}

En el presente trabajo de investigación se evaluaron 108 niños de 6 a 8 años, de ambos sexos distribuidos en dos grupos, de estudio y control; aquellos que consumían jugos de frutas industrializados y agua, respectivamente (Tabla 1). Se encontró que en el grupo de estudio conformado por los niños que consumían jugos de frutas industrializados, la media del pH salival era más ácido comparado con el grupo de control (Tabla 2).

Tabla 1. Distribución de la muestra según sexo de los escolares del I.E. Isabel La Católica.

\begin{tabular}{|c|c|c|c|c|}
\hline \multirow{2}{*}{$\begin{array}{l}\text { Variables } \\
\text { Sexo }\end{array}$} & \multicolumn{2}{|c|}{$\begin{array}{c}\text { Grupo de } \\
\text { estudio }\end{array}$} & \multicolumn{2}{|c|}{$\begin{array}{c}\text { Grupo de } \\
\text { control }\end{array}$} \\
\hline & $\mathrm{N}$ & $\%$ & $\mathrm{~N}$ & $\%$ \\
\hline Masculino & 18 & 36 & 33 & 56.9 \\
\hline Femenino & 32 & 64 & 25 & 43.1 \\
\hline
\end{tabular}

En el grupo de estudio prevaleció el pH ligeramente ácido, tanto en basal como a los 5 y 10 minutos. Así mismo se observó que 5 minutos después del consumo de la bebida, el $\mathrm{pH}$ disminuía ligeramente de 6.79 a 6.41 , pero se recuperaba a los 10 minutos, a $6.63 \pm 0.42$. Cabe resaltar que los valores del pH basal, es decir antes del consumo de las bebidas difería en ambos grupos, en el grupo de estudio se registró una media de 6.79 DS 0.47 , frente al grupo control que mostró una media de 7.11 DS 0.32 . (Tabla 2). El pH salival más bajo observado fue de 5.11 a los 5 minutos en el grupo de estudio y el más alto de 7.65 a los 10 minutos en el grupo control.

En el grupo de estudio hubo un mayor número de escolares con $\mathrm{pH}$ ligeramente acido comparado con los escolares del grupo control, mientras que en este último grupo, la mayoría presentó un $\mathrm{pH}$ neutral, es decir con valores de 7 a 7.4 (Tabla 3).

En el grupo de estudio, si hubo diferencia significativa entre el pH basal y a los 5 minutos, y entre 5 minutos $y$ de 10 minutos. Friedman $=0.001$. (Tabla 4) Al compararse los $\mathrm{pH}$ del grupo control y de estudio en los momentos no hay diferencia significativa como se puede ver en la y Figura 1. 
Tabla 2. Valores medios del pH salival de los escolares de la I.E. Isabel La Católica en los grupos de estudio y control según intervalos de tiempo.
Grupo de estudio
Grupo de control

\begin{tabular}{lcccc}
\hline \multicolumn{1}{c}{$\mathrm{pH}$} & Media & Desviación estándar & Media & Desviación estándar \\
\hline Basal & 6.79 & 0.47 & 7.11 & 0.32 \\
5 minutos & 6.41 & 0.43 & 7.12 & 0.31 \\
10 minutos & 6.63 & 0.42 & 7.18 & 0.40 \\
\hline
\end{tabular}

* Prueba de Friedman $p=0.001$

* Prueba de Friedman $p=0.11$

Tabla 3. Número y porcentaje de escolares de la I.E. Isabel La Católica en los grupos de estudio y control según el tipo de pH salival e intervalos de tiempo

\begin{tabular}{|c|c|c|c|c|c|c|c|c|c|c|c|c|}
\hline \multirow[b]{3}{*}{$\mathrm{pH}$} & \multicolumn{4}{|c|}{ Grupo de estudio } & & & \multicolumn{6}{|c|}{ Grupo de control } \\
\hline & \multicolumn{2}{|c|}{ Acido } & \multicolumn{2}{|c|}{ Neutral $^{* *}$} & \multicolumn{2}{|c|}{ Básico } & \multicolumn{2}{|c|}{ Acido } & \multicolumn{2}{|c|}{ Neutral $^{* *}$} & \multicolumn{2}{|c|}{ Básico } \\
\hline & $\mathrm{N}^{\circ}$ & $\%$ & $\mathrm{~N}^{\circ}$ & $\%$ & $\mathrm{~N}^{\circ}$ & $\%$ & $\mathrm{~N}^{\circ}$ & $\%$ & $\mathrm{~N}^{\circ}$ & $\%$ & $\mathrm{~N}^{\circ}$ & $\%$ \\
\hline Basal & 18 & 81.8 & 30 & 38.5 & 2 & 25.0 & 4 & 18.2 & 48 & 61.5 & 6 & 75.0 \\
\hline 5 minutos & 34 & 94.4 & 15 & 22.4 & 1. & 20.0 & 2 & 5.6 & 52 & 77.6 & 4 & 80.0 \\
\hline 10 minutos & 23 & 95.8 & 26 & 31.1 & 1. & 7.1 & 1 & 4.2 & 44 & 62.90 & 13 & 92.90 \\
\hline
\end{tabular}

${ }^{* *} \mathrm{pH}$ de 7 a 7.4

Tabla 4. Comparación del pH salival del grupo de estudio en escolares de la I.E. Isabel La Católica

\begin{tabular}{ccc}
\hline pH & \multicolumn{1}{c}{$p H$} \\
basal & vs & 5 minutos* \\
basal & vs $\quad 10$ minutos \\
5 minutos & vs $\quad 10$ minutos* \\
\hline & & \\
*Prueba de Friedman $p=0.001$ \\
Comparaciones múltiples de Dunn's $p<0.001$
\end{tabular}




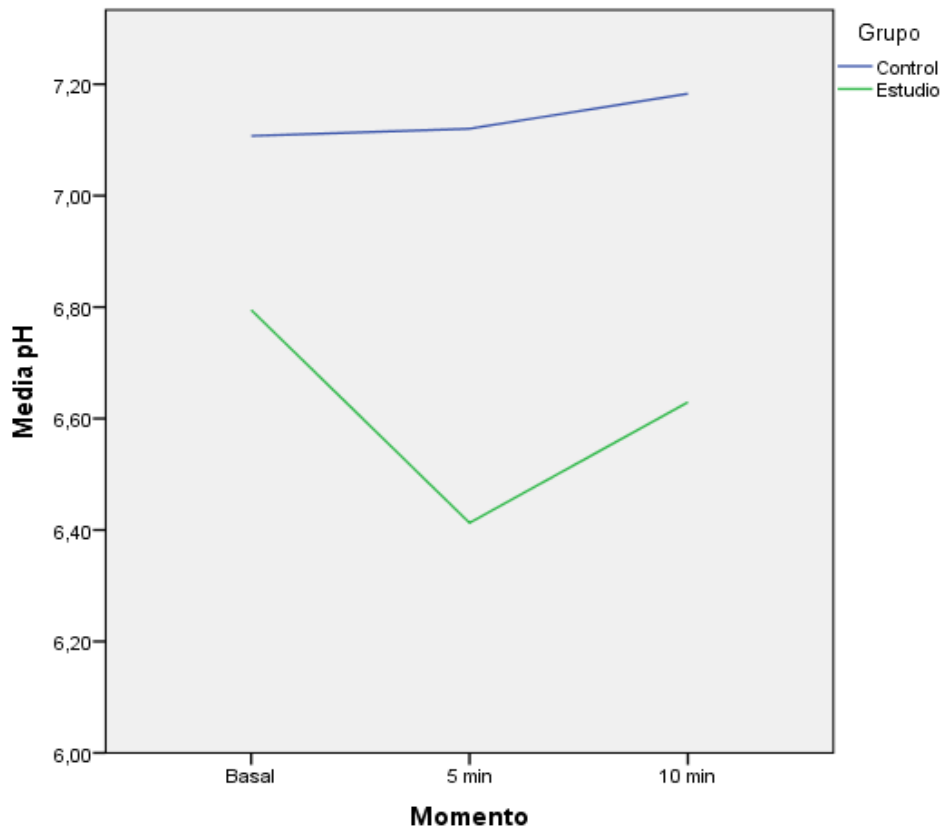

U de Mann, Whitney, W de Wilcoxon $p=0.000$

Figura1. Comparación entre los valores del pH basal a los 5 y 10 minutos del grupo de estudio y control en escolares de la I.E. Isabel La Católica

El Índice gingival del grupo de estudio fue de 0.62 y del control 0.35 . Entre el índice gingival y el pH salival del grupo de estudio y de control, también se aplicó la prueba de RhO de Spearman encontrándose diferencias sin valor estadístico $p>0.05$. Los valores máximos fueron 1.25 en el grupo de estudio y 1.75 en el grupo control.

Tabla 5. Índice Gingival Löe Silness en los escolares de la I.E. Isabel La Católica. 2016

Grupo estudio

\begin{tabular}{cccc}
\hline Media & Desviación estándar & Media & Desviación estándar \\
\hline 0.62 & .38 & 0.35 & .37
\end{tabular}

Grupo control 


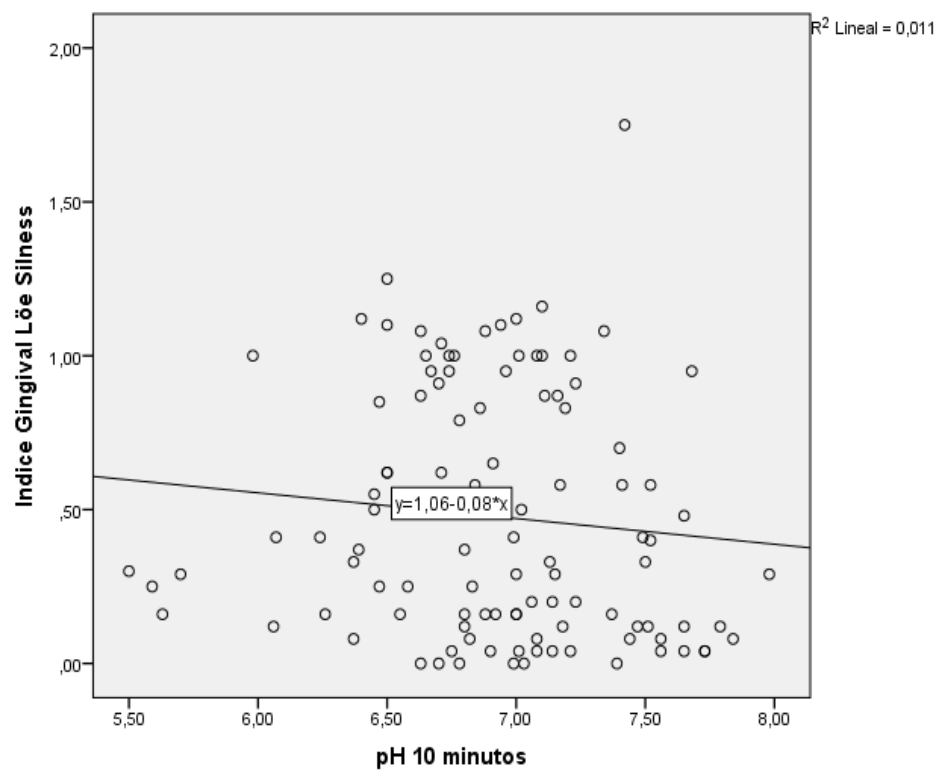

Figura 2. Índice Gingival y pH en escolares de la I.E. Isabel La Católica, a los 5 minutos

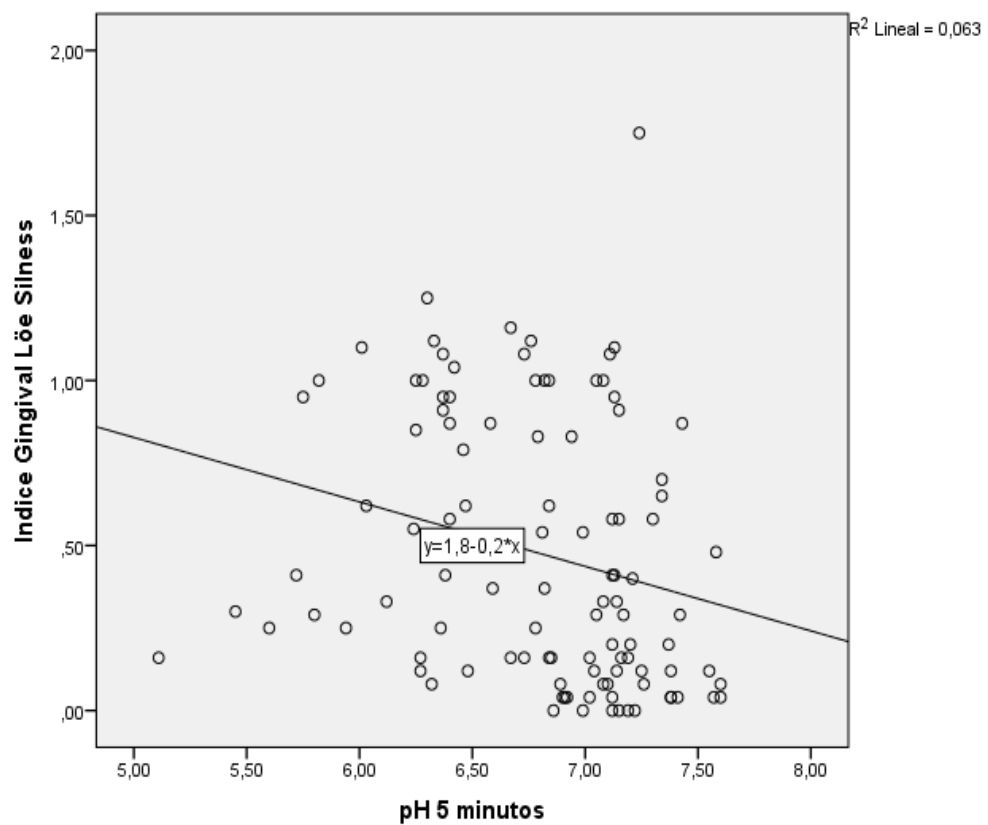

Figura 3. Índice Gingival y pH en escolares de la I.E. Isabel La Católica, a los 10 minutos 
Tabla 6. Correlaciones entre el Índice Gingival Löe Silness y los valores de pH en los tres momentos de los grupos de estudio y control de los escolares del I.E. Isabel La Católica.

\begin{tabular}{cccc}
\hline & \multicolumn{3}{c}{ Significancia bilateral (p-valor) } \\
\hline \multicolumn{1}{c}{ Correlación Rho de Spearman } & pH Basal & pH 5 min & pH 10 min \\
\hline Índice Gingival Löe Silness Grupo Control & 0.300 & 0.269 & 0.809 \\
\hline Índice Gingival Löe Silness Grupo Estudio & 0.258 & 0.952 & 0.128 \\
\hline
\end{tabular}

RhO de Spearman $p>0.05$

Respecto a la placa dental se encontró que tanto en el grupo de estudio como de control existe, correlación significativa entre Índice de higiene oral y el $\mathrm{pH}$, a los 5 minutos y a los 10 minutos, Rho de Spearman $p=0.01$

Al contrastar las variables higiene oral con gingivitis, tanto en el grupo de estudio como de control, existe correlación significativa, que indica que al aumentar la placa dental aumenta también el índice gingival, Rho de Spearman $p=0.01$

Analizando el índice gingival PMA, el grupo de estudio presentó medias de $17.72,10.36$ y 2.98 en la gingivitis papilar, marginal y adherida respectivamente, en contraposición a los valores del grupo control que fueron de $9.50,8.60$ y 0.00 en las respectivas gingivitis. Al aplicar la prueba de $U$. de Mann Whitney y W de Wilcoxin se obtuvo un $p=$ 0.033 en la gingivitis papilar entre ambos grupos y $p$ $=0.014$ en gingivitis de encía adherida

\section{DISCUSIÓN}

En este estudio se encontró un $\mathrm{pH}$ ligeramente ácido en el grupo de estudio, con una media de $6.41 \pm 6.43$, mayor a lo notificado por Goel et al.(11) quien encontró en su estudio realizado en un grupo de 39 niños entre 8 a 12 años de edad, un pH salival que variaba de 3.21 a 6.86 con un valor medio de 4.89 después del consumo de estos jugos de frutas. Con el consumo de bebidas carbonatadas, Goel encontró una media de $\mathrm{pH}$ salival de $5.47 \pm 0.78$

En el presente estudio se evidencia que en el grupo que consumían los jugos de frutas industrializados, el $\mathrm{pH}$ salival era más bajo comparado con el grupo de niños que habitualmente llevaban botellas de agua. Similar a lo reportado por Sp Shetgar et al. ${ }^{(10)}$ quienes encontraron una caída del $\mathrm{pH}$ salival después del consumo de diferentes tipos de jugos de frutas, al contrastar la medición basal con los valores obtenidos a los $0,10,30$ y 60 minutos, llegando a un $\mathrm{pH}$ de 3,12 . Observaron también que el pH fue más bajo en la medición realizada inmediatamente después de ingerir los jugos de frutas.

Con respecto a la relación del $\mathrm{pH}$ salival y la condición gingival, en el presente estudio no se encontró correlación entre estas variables, en los tres momentos de la medición. En el grupo control se encontró una correlación inversa entre el Índice Gingival de Lôe Silness y el pH salival a los 5 minutos. En el grupo control la media del pH fue de $7.12 \mathrm{con}$ un mínimo de 5.45. A diferencia de Ahlam T. et al(17) ,quienes encontraron que las medias del $\mathrm{pH}$ salival, en una población de niños de 4 a 5 años, se elevaron con el aumento de la severidad de la inflamación gingival, tanto en el grupo de estudio como de control, aunque sin diferencias estadísticamente significativas. El más alto porcentaje de niños (65\%) tuvo un pH igual a 7 . Los resultados de este estudio coinciden con los reportados por Ysla (2006) (15), quien trabajo con niños de 6 a 8 años, con síndrome de Down y un grupo control.

Al contrastar los valores del Índice de Higiene Oral y el pH salival se observó que había una correlación estadísticamente significativa, a los 5 minutos y a los 10 minutos, en ambos grupos, se encontró que el pH salival disminuía cuando se incrementaban los valores del Índice de placa $(p=0.01), \quad$ similar a lo informado por Ahlam T.et al (17).

Cabe resaltar que la prevalencia de gingivitis en los escolares examinados fue muy alta, llegando a un $90 \%$, una cifra que coincide con lo reportado por Juarez ${ }^{(12)}$ que encontró un $70 \%$ en escolares de 4 a 6 años con alteraciones gingivales, Sánchez (13) que obtuvo un $93,1 \%$ de gingivitis en una población de 102 niños chilenos y Cárdenas ${ }^{(14)}$ que encontró un $90.77 \%$ en niños de 6 años.

Los resultados de este estudio muestran que al ingerir los jugos de frutas industrializados desciende el ph salival lo que se corrobora con estudios anteriores; es 
importante considerar que el consumo de estos jugos en las escuelas puede ser alto y frecuente y si a esto sumamos que al aumentar la presencia de placa dental el pH salival también disminuye, estaríamos frente a dos factores que estarían poniendo en serio riesgo la salud bucal de los niños. Aun cuando en este estudio no se encontró evidencia que el pH salival por el consumo de jugos de frutas industrializado esté relacionado con la salud gingival; se sugiere realizar nuevos estudios en el cual se trabaje con una muestra mayor y se incluyan otras variables relacionadas al consumo de bebidas que los escolares consuman habitualmente, como las bebidas carbonatadas.

Se llegó a las siguientes conclusiones: No hay correlación entre el índice gingival de Lôe Silness y los valores del $\mathrm{pH}$ salival. El pH salival de los niños de 6 a 8 años que consumen jugos de frutas industrializados es ligeramente ácido en los intervalos basal, de 5 y 10 minutos. Los valores del pH salival aumentan a medida que decrece el índice gingival en el grupo que consume agua. El $\mathrm{pH}$ salival se hace más ácido a medida que aumentan los valores del Índice de Higiene oral. Los niños de la muestra de estudio presentan un índice gingival moderado.

Se recomienda educar a los padres y familiares de los niños sobre los efectos del $\mathrm{pH}$ de los jugos endulzados que llevan en sus loncheras. Los odontólogos y pediatras debiesen advertir del daño que causa el $\mathrm{pH}$ salival ácido sobre el esmalte dentario.

\section{Agradecimientos}

- Al Dr. Rafael Morales Vadillo por su apoyo en el análisis estadístico

- Al Lic. Areovisto Bustamante Sempertegui, Director del I.E. Isabel La Católica.

- Profesoras de la I.E. Isabel La Católica, especialmente a Miss Manuela Aspajo y a las profesoras de $1^{\circ}$ y $2^{\circ}$ de primaria.

- A las estudiantes de la asignatura de Periodoncia II: Gianella Ramírez Jeri y Katnerine Solís Anyosa.

Contribuciones de autoría: RYC, diseño el estudio. RYC y MPV recopilaron y procesaron los datos y redactaron el artículo. Ambas autoras aprobaron la versión final del artículo.

Fuente de financiamiento: El estudio fue financiado por los autores.

Conflicto de intereses: Los autores declararon no tener conflictos de interés.

\section{REFERENCIAS BIBLIOGRÁFICAS}

1. Ruilova Carrión Camilo Eduardo, León Arbulú Diana Cecilia, Tay Chu Jon Lidia Yileng. Potencial erosivo de jugos naturales, jugos industrializados y gaseosas: Revisión de Literatura. Rev. Estomatol. Herediana [Internet]. 2018 Ene [citado 2018 may 29] ; 28(1): 56-63. Disponible en: http://www.scielo.org.pe/scielo .php?script=sci_arttext\&pid=S1019-43552018000100007\&lng=es.

2. Aykut-Yetkiner A1, Wiegand A, Ronay V, Attin R, Becker $\mathrm{K}$, Attin $\mathrm{T}$. In vitro evaluation of the erosive potential of viscosity-modified soft acidic drinks on enamel. Clin Oral Investig. 2014 Apr;18(3):769-73.

3. Reddy A, Norris F. D, Momani S, Waldo B, Ruby J. The $\mathrm{pH}$ of beverages in the United States. Jada 2016;146(4):255-263.

4. Vitorino R, Calheiros-Lobo MJ, Duarte JA, Domingues $P$, Amado F. Salivary clinical data and dental caries susceptibility: is there a relationship? Bull Group Int Rech Sci StomatolOdontol 2006; 47(1):27-33.

5. Guías Prácticas de Estomatología. Editorial Ciencias Médicas. La Habana, 2007: 23 - 47.

6. Banderas, J. A; González, M. Saliva y cavidad bucal. Parte II: Proteínas salivales: funciones biológicas en el mantenimiento de la homeostasis bucal. Pract Odontol 2007; 15 (7): 13-20.

7. Radha G, Swathi V, Jha Abhishek. Assessment of salivary and plaque $\mathrm{pH}$ and oral health status among children with and without intellectual disabilities. Journal of Indian society pedodontic and preventive dentistry. 2016; 34 (3): 257-261.

8. Contero Mejia, Paola, \& Cabrera Dávila, Ma. José. (2018). Correlación entre ph salival y caries dental en pacientes con síndrome de down que acuden a la fundación asistencial armada nacional, Guayaquil, Ecuador-2018(Citado 2018 abril 26) ;14(61):15-20. Disponible en: http://scielo.sld.cu/scielo.php? script=sci_arttext\&pid=S1990-86442018000100002\&lng=es\&tlng=es.

9. Barrios C, Vila V, Martinez S, Tutuy A. Ph Salival como factor asociado a la caries dental investigación. 2017; 6(1): 13-17.

10. Sp Shetgar, Umesh Kemparaj, Sangeeta Chavan,Rahul Patel. Effect of Fresh Fruit Juices on Salivary pH: A Randomized Controlled Trial. International Journal of Oral Health and Medical Research. 2017;3(5): 28-32.

11. Goel Isha, Navit S., Singh Mayall Sandeep, Rallan Mandeep, Navit Pragati, Chandra Sneha. Effects of Carbonated Drink \& Fruit Juice on Salivary $\mathrm{pH}$ of Children: an in vivo study. International Journal of Scientific Study. 2013;1 (3): 60 - 9.

12. Juárez-López María Lilia Adriana, Murrieta-Pruneda José Francisco, Teodosio-Procopio Elizabeth. Prevalencia y factores de riesgo asociados a enfermedad periodontal en preescolares de la Ciudad de México. Gac. Méd. Méx [revista en la Internet]. 2005 Jun [citado 2018 Mar 20] ; 141( 3 ): 185-189. Disponible en: http://www.scielo.org.mx/scielo.php? script=sci_arttext\&pid=S001638132005000300003\& ng=es. 
13. Zaror Sánchez C., Muñoz Millán P., Sanhueza Campos A.. Prevalencia de gingivitis y factores asociados en niños chilenos de cuatro años. Av Odontoestomatol [Internet]. 2012 Feb [citado 2018 Mar 29] ; 28( 1 ): 33-38. Disponible en: http://scielo.isciii.es/scielo.php?script=sci_arttext\&pid =S0213-12852012000100005\&lng=es..

14. Cárdenas Espinoza C., Romero Saavedra M., Giacaman Sarah RA. Evolución de la prevalencia de caries y gingivitis en niños de 6 y 12 años de Peralillo, VI Región, entre el año 2000 y el 2010. Rev. Clin. Periodoncia Implantes, Rehabil Oral. 2011; 4 (3): 102 $-108$.
15. Ysla Chee Rosa Elizabeth. Impacto en salud oral asociada con el pH salival en niños con Síndrome de Down de centros de Educación Especial de Lima, 2005 [ Tesis Doctoral en Salud Publica]. Lima, Peru. Universidad Nacional Federico Villarreal. 2006.

16. Roopavathi KM, Gopal SV, Pushpalatha G et. al. Oral health status and prevalence of gingivitis among 6-14 years old school children in Tumkur city - a cross sectional study. Int J Health Sci Res. 2015; 5(3):200204

17. Al Mashhadani Ahlam T, Al-obaidi Wesal. The $\mathrm{pH}$ of stimulated saliva in relation to the oral health status among children an adults. J. College Dentistry. 2005; 17(1) : 89-91. 